\title{
Mortalidad infantil como indicador de desigualdad del sistema de salud chileno
}

\author{
Andrea Morales
}

Universidad de Chile

\section{Resumen}

Este trabajo busca documentar la distribución de la Tasa de Mortalidad Infantil (TMI) a nivel comunal y su relación causal a factores socio-económicos, demográficos y del sistema de salud. Se utilizaron las estadísticas vitales disponibles en el Departamento de Estadísticas e Información de Salud (DEIS) y características comunales disponibles en el Sistema Nacional de Información Municipal (SINIM), desde el año 2001 al 2011, para las 345 comunas chilenas. Se construyeron indicadores de desigualdad para mortalidad infantil e ingresos en salud municipal per cápita para cada año y se evaluó su correlación y evolución en el período. Utilizando modelos de datos de panel se evaluó la asociación de la tasa de mortalidad infantil a características socioeconómicas, sanitarias y de recursos municipales. Los resultados obtenidos muestran que mientras la mediana nacional de TMI por comunas se ha mantenido estable, la desigualdad a nivel comunal en este indicador ha ido en aumento. Mayor tasa de pobreza, menor nivel educacional de la población, no contar con Servicios de Atención Primaria de Urgencias (SAPU), menor inversión en salud municipal y mayor distancia al hospital base de referencia, resultan ser las principales características comunales que generan mayor mortalidad infantil en distintos modelos evaluados, con nivel de confianza sobre el $95 \%$.

Palabras clave: Mortalidad infantil, salud pública, equidad en salud.

Infant mortality as an indicator of inequality in the Chilean health system

\begin{abstract}
This work seeks to document the distribution of Infant Mortality Rate (IMR) at the community level and its causal relationship to socio-economic, demographic and health system factors. Vital statistics available from the Department of Health Statistics and Information (DEIS) and community features available on the National Municipal Information System (SINIM) were used from 2001 to 2011, for all of the 345 chilean comunes. Inequality indicators for infant mortality and income per capita in municipal health were built for each year and their correlation and evolution were evaluated during the period. The correlations of infant mortality rate to socioeconomic characteristics, health and municipal resources were evaluated using panel data models. The results show that while the national median of TMI by municipalities has been stable, inequality at the community level in this indicator has increased. The main communal features that generate higher infant mortality in different models tested, with confidence level of 95\%, are higher rates of poverty, lower educational level of the population, no Primary Care Services of Emergency, poor municipal investment in health and greater distance from the base reference hospital.
\end{abstract}

Keywords: Infant mortality, public health, health equity.

*Dirección de correspondencia [Correspondence

address]: Andrea Morales, Universidad de Chile

E-mail: dra.a.morales@gmail.com 


\section{Mortalidad infantil como indi- cador de desigualdad del siste- ma de salud chileno ${ }^{1}$}

Los indicadores utilizados para medir el estado de salud de una población constituyen indicadores líderes de desarrollo social, dada su estrecha asociación a condiciones socio-económicas (Castañeda, 1985; Silva y Durán, 1990; Jiménez y Romero, 2007; Pérez et al., 2012). En particular, la tasa de mortalidad infantil (TMI), definida como el número de muertes de menores de 1 año por cada 1000 nacidos vivos, es considerada universalmente un reflejo del estado de salud de la población en su conjunto y de las condiciones socio-económicas en las que ésta se desarrolla (Silva y Durán, 1990).

Sin embargo, los factores económicos no logran explicar por sí solos las variaciones que esta tasa experimenta entre distintos países o regiones, o incluso en su evolución histórica en un mismo territorio. Es por esto que se ha postulado que los factores económicos propiamente tales, como ingresos fiscales, empleo y salario, no constituirían el determinante central en la reducción de la mortalidad infantil (Castañeda, 1985; Pérez et al., 2012). Así, una tasa elevada de mortalidad infantil revela deficiencias en el entorno físico y socioeconómico, relacionadas a la nutrición, educación, saneamiento ambiental y cuidados de salud de una población. Los países de América Latina que han alcanzado altos niveles de desarrollo en indicadores sanitarios, cercanos a los de países desarrollados, comparten la existencia de una fuerte intervención estatal en este proceso, a través del desarrollo de políticas públicas sanitarias, enfocadas principalmente en programas de salud materno infantil, y políticas sociales relevantes (Jiménez y Romero, 2007).

En Chile, estas intervenciones sistemáticas, permitieron disminuir sostenidamente la mortalidad infantil desde 136,2 por 1000 nacidos vivos en la década del '50, a 7,4 el año 2010, a pesar de los períodos de recesiones económicas experimentados durante esas décadas (Castañeda, 1985; Jiménez de la Jara, 2001, Szot, 2002; Jiménez y Romero, 2007; CELADE., 2009; Ministerio De Salud Gobierno De Chile, 2010). Para el año 2010, Chile ocupaba la segunda menor mortalidad infantil de la región, detrás de Cuba (TMI de 5 por cada 1000 nacidos vivos ${ }^{2}$ ) y superaba a otros países de la OC-

\footnotetext{
${ }^{1}$ Esta investigación se produjo en el contexto de una tesis para optar al grado de Magíster en Políticas Públicas en la Universidad de Chile. El director de esta tesis fue Oscar Landerretche Moreno, profesor de la Facultad de Economía de la Universidad de Chile.

${ }^{2}$ Cuba, a diferencia de Chile, cuenta con despenalización del aborto sin restricciones causales (Center for Reproducti-
}

DE, como México y Turquía (Sistema De Información De Tendencias Educativas En América Latina, 2013).

Pese a que las cifras nacionales promedios han sido muy auspiciosas, permitiéndonos cumplir hasta ahora los compromisos internacionales adquiridos en la Declaración del Milenio, estas no revelan las disparidades que pueden observarse a lo largo de nuestro territorio (Chile, 2005). ¿Es Chile un país equitativo en cuanto al acceso, oportunidad y calidad de la atención de salud? ¿El sistema de salud chileno considera los factores determinantes del estado de salud de la población, en relación a las diferencias sociodemográficas y económicas existentes, para hacer frente a problemas de desigualdad en salud? ¿Cómo se refleja esto en nuestros indicadores sanitarios?, en especial en la Tasa de Mortalidad infantil, que además de su relevancia para el desarrollo nacional, es sin dudas un indicador de igualdad de oportunidades básicas.

Preguntas como estas han sido planteadas por diversos autores y organizaciones en los últimos años, haciendo un llamado a abandonar la "tiranía de los promedios" que puede llevar a ocultar las disparidades entre los diferentes sectores que conforman una sociedad (Salud, 2003; Kliksberg, 2005). Una aproximación para resolver estas preguntas pasaría, sin duda, por modificar la unidad de análisis desde el nivel nacional, clásicamente reportado, a la menor dimensión territorial con datos disponibles.

El primer informe emitido por el Chile (2005) sobre el cumplimiento de los Objetivos del Milenio, si bien señala que el país presenta una tendencia favorable en la reducción de la mortalidad infantil, señala también que existen marcadas diferencias en esta tasa a lo largo del territorio, observándose que esta es más alta que el promedio nacional en las comunas de bajos niveles de ingresos. Sin embargo, este informe no detalla las tasas observadas a nivel comunal y, por tanto, no revela la magnitud del problema. Más aún, a pesar de mencionar estas diferencias, al analizar los principales desafíos para la mantención de la tendencia decreciente en la TMI nacional, no se plantean intervenciones sobre las comunas que persistirían con tasas elevadas; más bien, plantea "enfocar los esfuerzos a grupos específicos de alta mortalidad comparada con la de países desarrollados, y en los cuales es posible tener un impacto significativo en términos de mortalidad y morbilidad". Estos grupos serían los prematuros y los nacidos con malformaciones congénitas, por lo cual, consecuentemente, se plantearon intervenciones específicas relacionadas a cuidados neonatales, obstétricos y perinatales, con un alto costo de oportunidad y sin considerar intervenciones para

ve Rights, 2007). 
mitigar las diferencias comunales mencionadas, lo cual podría contribuir a agravar estas disparidades (Chile, 2005).

Por otra parte, si actualmente las mayores tasas se reconocen en comunas de menores ingresos ¿cabe plantear entonces, que los factores involucrados en estas diferencias sí serían de tipo económico, toda vez que los programas específicos de salud maternoinfantil se han llevado a cabo por décadas a lo largo de todo el territorio?

Conocer la situación de las comunas que persisten rezagadas en cuanto a mortalidad infantil y conocer los factores que pudiesen explicar este rezago, permitiría diseñar políticas públicas apropiadas a la reducción de la mortalidad infantil en aquellas comunas que se encuentran lejos de los buenos estándares nacionales. En este sentido, el presente estudio pretende revelar la evolución de la TMI a nivel comunal en los últimos 11 años y evaluar los factores socio-económicos, demográficos y del sistema de salud, que puedan dar cuenta de las diferencias que se observen entre las comunas chilenas, con el fin de contribuir al apropiado abordaje de las políticas públicas sanitarias que contribuyan hacia la igualdad de oportunidades básicas en niños, altamente valorada por la sociedad.

\section{Impacto de la equidad en salud}

Actualmente, los países desarrollados centran sus esfuerzos en lograr una distribución equitativa en la asignación de recursos a Salud. Sin embargo, se han planteado distintas definiciones de equidad en salud (Sánchez y Albalá, 2004; Raña et al., 2007; Urriola, 2007):

1. lograr que el gasto per cápita sea igual para toda la población,

2. que cada individuo reciba el volumen de recursos requeridos para satisfacer sus necesidades, o

3. vincular estos recursos per cápita al cumplimiento de metas de acceso y cobertura.

La relevancia de centrar el enfoque de políticas públicas hacia el avance en equidad en Salud radica en la amplia evidencia de la influencia que ejerce la pobreza y la desigualdad de ingresos sobre los indicadores sanitarios de una población (Starfield y Shi, 2002; Starfield et al., 2005; Organización Panamericana De La Salud, 2003). Así, se ha observado que países con menor desigualdad en la distribución del ingreso, logran mejores resultados sanitarios, principalmente, gracias a la implementación de políticas sanitarias en niveles locales (Organización Panamericana De La Salud, 2003, 2005).

La Organización Panamericana de la Salud (OPS), en un documento de posición sobre la necesidad de renovación de la Atención Primaria de Salud (APS) publicado el 2005, hace referencia a que América se encuentra en un contexto de empeoramiento global de las inequidades en salud (Pan American Health Organization, 2002; Organización Panamericana De La Salud, 2005). A modo de ejemplo, cita que durante la década del 2000 "el 60 por ciento de la mortalidad materna se produjo en el 30 por ciento de la población más pobre de los países; y, que en algunos de ellos, la brecha en la esperanza de vida entre los más ricos y los más pobres llegó a ser de casi veinte años" (Organización Panamericana De La Salud, 2005).

En Chile se han obtenido grandes avances desde la segunda mitad del siglo XX, con respecto a los tradicionales indicadores sanitarios: esperanza de vida al nacer, mortalidad materno-infantil y mortalidad general, alcanzando indicadores sanitarios cercanos a los de países desarrollados (Jiménez de la Jara, 2001; Szot, 2002; Valdivia, 2006; Albala et al., 1993). Sin embargo, estos indicadores presentan heterogeneidad al evaluarlos por distintos niveles de organización geopolítica, lo cual evidenciaría la desigualdad en el estado de salud de la población chilena (Sánchez y Albalá, 2004; Vargas y Wasem, 2006).

Sánchez y Albalá (2004) encontraron una relación negativa entre mortalidad general y nivel de ingreso por hogar o comuna. Similares hallazgos se han obtenido en relación a mortalidad infantil. Un estudio publicado por Donoso (2004), reveló la desigualdad existente en la Mortalidad infantil entre las comunas de la provincia de Santiago, demostrando que las variables económicas de Ingreso, Pobreza y Desempleo, se asocian significativamente a este indicador.

Kaempffer y Medina (2006) utilizando las estadísticas sanitarias del año 2003, estudiaron la Tasa de Mortalidad infantil a nivel regional, provincial y comunal, encontrando que este indicador no presentaba grandes diferencias a nivel regional (rango entre 7,5 para la III y Región Metropolitana, a 10,1 para la XI Región), pero que, al evaluar por provincias se observaba mayor dispersión (rango entre 6,1 para la provincia de Curicó y 14,3 para Arauco). Los autores destacan que a nivel comunal ${ }^{3}$, un $11 \%$ duplicaban el promedio nacional de Mortalidad Infantil y 1,8\% lo cuadruplicaban; mientras que, en

\footnotetext{
${ }^{3}$ Para 336 comunas.
} 
un cuarto de las comunas no se registraron muertes en menores de un año. A nivel regional, los autores obtuvieron una correlación significativa entre Mortalidad Infantil y desempleo $(\mathrm{rho}=0,85)$, desnutrición materna durante el embarazo $(\mathrm{rho}=0,63)$, bajo peso de nacimiento (rho $=0,58)$, disponibilidad de recursos técnicos en el Sistema Nacional de Servicios de Salud (SNSS) (rho $=0,52)$, atención profesional del parto $(\mathrm{rho}=0,50)$, escolaridad $($ rho $=0,40)$, residencia urbana $($ rho $=0,35)$ y alfabetismo (rho $=0,33$ ). A nivel nacional, se correlacionó positivamente a bajo peso de nacimiento, baja escolaridad materna, edad materna y número de hijos mayor o igual a 10 y negativamente, a peso de nacimiento mayor o igual a 3 kilos, escolaridad universitaria materna y madre perteneciente a población económicamente activa (Vargas y Wasem, 2006). Los autores destacan como limitación del estudio, los distintos períodos de tiempo del que fueron obtenidos los datos: estadísticas de las décadas del ?80 y ?90 para las variables explicativas y cifras del año 2003 para Mortalidad Infantil, por la dificultad de contar con datos sociodemográficos y epidemiológicos actualizados (Kaempffer y Medina, 2006).

Recientemente, Flores y Cerda (2012) midieron la evolución de la desigualdad en mortalidad infantil en comunas de distinto nivel socioeconómico de la Región Metropolitana (caracterizado mediante el índice de pobreza $\mathrm{CASEN}^{4}$ ), entre los años 1990 y 2006, calculando riesgos atribuibles y razón de tasas. Los autores concluyen que la desigualdad en la TMI habría disminuido entre comunas de la Región Metropolitana, pero persistiendo al año 2006 importantes brechas (Flores y Cerda, 2012). Finalmente, Castro (2013) encuentra una disminución progresiva, hasta la eliminación, de la asociación de mortalidad infantil a nivel socioeconómico de la población comunal (medido a través de un Índice socioeconómico comunal, construido en base al ingreso autónomo per cápita del hogar, a partir de datos de la encuesta CASEN), en una muestra de comunas entre los años 1990 a 2010.

Los antecedentes presentados revelan importantes avances en el conocimiento sobre la desigualdad en salud en Chile y, específicamente, en Mortalidad infantil. Sin embargo, en la actualidad es necesario ahondar en la medición de esta desigualdad y en el análisis de los posibles factores sociales, económicos y demográficos implicados, con el fin de reenfocar la atención sobre el cumplimiento de los Objetivos de Desarrollo del Milenio, desde las prometedoras cifras nacionales hacia las deficiencias que persistan en niveles subnacionales y locales, en los cuales

\footnotetext{
${ }^{4}$ Encuesta de Caracterización Socioeconómica nacional, realizada por el Ministerio de Desarrollo Social de Chile.
}

la atención primaria de salud (APS) juega un rol central $^{5}$.

\section{Metodología}

A partir de los datos publicados por el Departamento de Estadísticas e Información de Salud (DEIS), se obtuvo el reporte de la TMI comunal desde el año 2001 al 2011 en las 345 comunas del país ${ }^{6}$. Con estos datos se evaluó la evolución de la TMI a nivel comunal en el período, graficándolos en mapas territoriales y construyendo distintos indicadores de desigualdad entre comunas para cada año. También se construyeron indicadores de desigualdad en ingreso de salud municipal per cápita, evaluando su asociación a desigualdad en mortalidad infantil.

Del Sistema Nacional de Información Municipal (SINIM), dependiente de la Subsecretaría de Desarrollo Regional y Administrativo (SUBDERE), se obtuvieron características demográficas y socioeconómicas de la población comunal, características presupuestarias y de disponibilidad de recursos en salud municipales, las cuales pudieran considerarse como posibles predictores de TMI.

Se evaluó la correlación entre estas variables y la TMI comunal, por cada año de análisis, a través de análisis de correlación múltiple no paramétrico (coeficiente de Spearman). Las variables que resultaron relacionadas a mortalidad infantil (o por sustento en la literatura), fueron evaluadas en modelos de regresión con datos de panel. Finalmente, se realizó un análisis de componentes principales con las variables explicativas, para la identificación de perfiles comunales en relación a la mortalidad observada para el período.

\section{Resultados}

\section{Caracterización de las comunas y su relación a Mortalidad Infantil}

El Gráfico 1, muestra la evolución de la mediana y recorrido intercuartílico de la TMI a nivel de

\footnotetext{
${ }^{5}$ Según datos de FONASA, el año 2012 la APS en Chile otorgaba cobertura de salud a cerca de 13 millones de habitantes, lo cual equivale aproximadamente, a $75 \%$ de la población chilena (33). La APS es de administración mayoritariamente municipal (96,3\% de las comunas administraban un sistema de salud municipal el año 2011) y financiamiento compartido con el Gobierno Central.

${ }^{6}$ A partir del año 2004, cuando se crea la comuna de Alto Hospicio en la I Región de Tarapacá.
} 
las comunas chilenas, entre los años 2001 y 2011. Se observa que mientras la mediana de Mortalidad infantil se ha mantenido estable (en torno a 7,2 por 1000 nacidos vivos), la dispersión del indicador no ha mostrado la misma estabilidad, principalmente por la mayor variación que ha experimentado el percentil 25 de la distribución entre los años 2001 y 2008, manteniéndose posteriormente en cero.

Un 57,4\% ( $\mathrm{n}=198)$ de las comunas no presentaron mortalidad infantil en al menos uno de los once años y $3,5 \%(n=12)$ no presentaron mortalidad infantil en todo el período. Al evaluar la distribución de habitantes y nacimientos a nivel comunal, se observa que estas comunas presentan significativamente menor número de habitantes y nacimientos que las comunas con defunciones infantiles. Así, el recorrido intercuartílico del tamaño poblacional en estas comunas equivale al percentil 25 de la población en el total de comunas en cada año, al igual que lo observado para la distribución de la TMI.

En cuanto a la localización regional, las comunas sin defunciones infantiles pertenecen a regiones extremas del país (Magallanes, Aysén, Arica y Parinacota y Tarapacá); mientras que, las comunas que presentaron mayor mortalidad infantil, por sobre la mediana nacional de cada año, se ubican en las regiones de La Araucanía, Atacama, Los Ríos y Coquimbo.

\section{Características Socioeconómicas}

A nivel comunal, entre el año 2001 y 2011 la mediana de población que vive bajo la línea de pobreza disminuyó en $38 \%$, con moderada variabilidad intercomunal en ambas años (coeficiente de variación ¿0,4). La escolaridad promedio de la población aumentó en $7 \%$, manteniendo una baja variabilidad entre comunas. Sin embargo, el retiro de la enseñanza media aumentó en 19\%, con una alta variabilidad intercomunal en ambos años (coef. de variación: 0,8$)$.

\section{Características Demográficas}

La mediana de población comunal aumentó en $90 \%$, manteniendo una alta variabilidad intercomunal (sobre 1,5). La Tasa de Natalidad disminuyó un $13 \%$ en el período, mientras que la proporción de niños menores de 2 años beneficiarios del sistema de salud municipal ${ }^{7}$ aumentó en $30 \%$, con baja variabilidad entre las comunas en ambos casos.

\footnotetext{
${ }^{7}$ Población beneficiaria: Población inscrita en servicios de atención primaria municipal, validada por FONASA.
}

\section{Urbanización}

La cobertura de viviendas con alcantarillado aumentó en $20 \%$ entre 2001 y 201; mientras que el porcentaje de población que cuenta con acceso a agua potable se mantuvo constante, con una mediana de cobertura de $80 \%$. Ambas variables presentaron baja variabilidad intercomunal. La proporción de población urbana aumentó en $10 \%$ y la densidad de población en $5 \%$, ambas con alta variabilidad intercomunal.

\section{Recursos Financieros y Sanitarios Municipa- les}

La dependencia al Fondo Común Municipal (FCM) sobre los ingresos propios aumentó en $3 \%$, manteniendo una variabilidad de $40 \%$. El gasto en asistencia social per cápita aumentó en $88 \%$, con un aumento de la variabilidad entre comunas (de $1,7$ a 2,4$)$.

La cobertura de salud municipal ${ }^{8}$ aumentó en $21 \%$ entre los años 2001 y 2011, manteniendo una baja variabilidad entre las comunas. El gasto en salud municipal por población inscrita ${ }^{9}$ aumentó en $100 \%$, manteniendo una variabilidad intercomunal entre 30 y $40 \%$. Este aumento en el gasto podría explicarse por la considerable inyección de recursos que experimenta el sector desde el año 2005 a la fecha, con la implementación del Régimen de Garantías Explícitas en Salud (GES), componente central de la Reforma de Salud, y que en sus primeros años estuvo destinado en gran medida a financiar las prestaciones garantizadas en el sistema de salud municipal.

Por el contrario, la mediana de inversión en salud municipal por población inscrita ${ }^{10}$ se mantuvo en niveles muy bajos, pero aumentó considerablemente la variabilidad entre comunas (de 1,2 a 9,2). Esto se relaciona con la baja disponibilidad de centros de atención primaria (urbanos y rurales) por inscrito en el sistema de salud municipal. En el caso de los consultorios y postas de salud rural, la tasa de centros disminuyó de 0,3 a 0,2 por cada 1.000 inscritos y la tasa de servicios de atención primaria de urgencias (SAPU) se mantuvo en una mediana de 0 por cada 10.000 inscritos, lo cual muestra la baja creación de nuevos centros de salud municipal en relación al aumento en la cobertura del sistema. Ambos indicadores presentan una alta variabilidad

\footnotetext{
${ }^{8}$ Corresponde a la proporción de población comunal afiliada al sistema de salud público e inscrita en algún centro de salud municipal

${ }^{9}$ Incluye los aportes ministeriales y municipales al sector

${ }^{10}$ Destinada a infraestructura y equipamiento para la atención primaria
} 
Figura 1: Evolución de la Mortalidad Infantil Comunal. Chile, 2001 - 2011

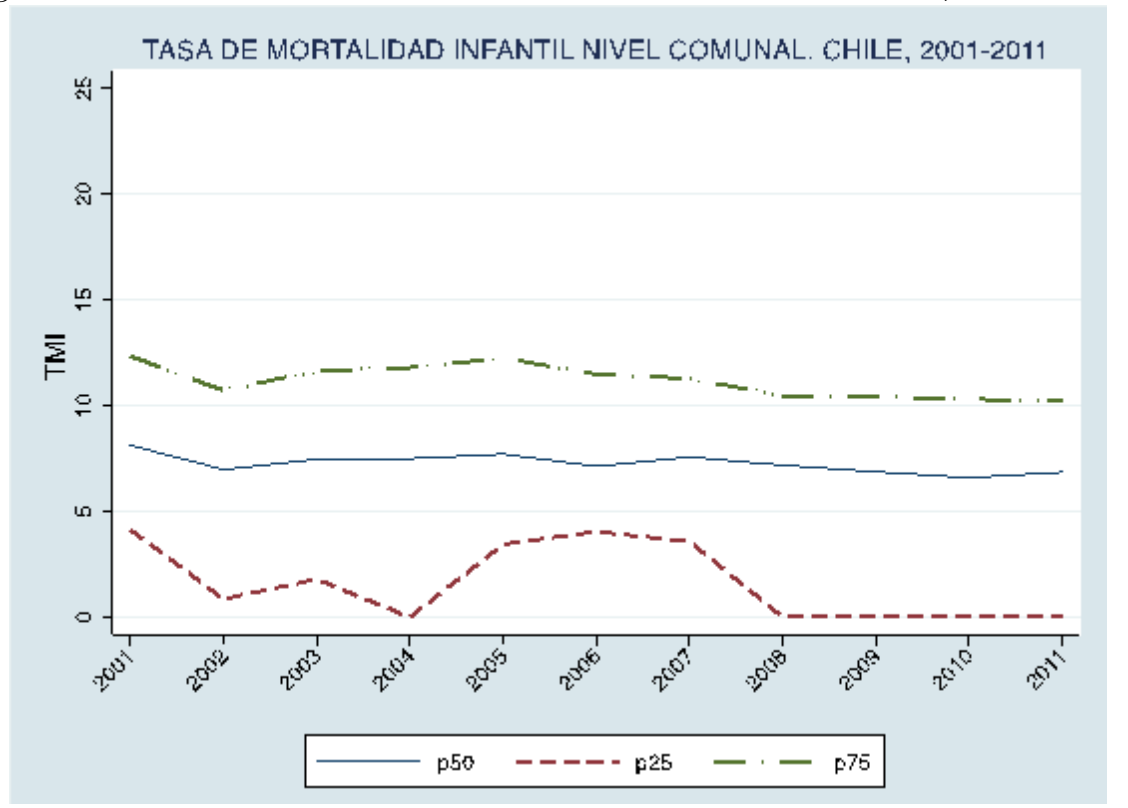

entre comunas en cada año, superiores al $100 \%$.

La tasa de médicos en atención primaria municipal por cada 1.000 habitantes presentó un crecimiento desde 0,2 a 0,3 el 2011, manteniéndose muy por debajo de la tasa de médicos disponibles en todo el sector público del país (1,1 por 1000 beneficiarios FONASA) y del promedio de países de la $\operatorname{OCDE~(3,1~por~} 1000$ el año 2009) (34-35). Sin embargo, la variabilidad del indicador entre comunas disminuyó de 1,5 a 0,8 , lo cual hablaría de un avance hacia una distribución más homogénea de los médicos en la atención primaria.

En cuanto a la distancia al hospital base de referencia, la situación se mantuvo constante, manteniendo una alta dispersión entre las comunas (coeficiente de variación de 2,0), observándose que mientras la mediana de distancia se mantuvo en $16 \mathrm{~km}$, persisten comunas que se encuentran a $700 \mathrm{~km}$ del centro de derivación.

\section{Análisis de correlación múltiple}

Con el fin de obtener una aproximación inicial a posibles relaciones entre características comunales y Mortalidad infantil, se realizó un análisis de correlación no paramétrico entre las variables comunales descritas y la TMI comunal.

Al incluir el total de comunas con datos disponibles, la mortalidad infantil presenta una correlación positiva a la tasa de pobreza, decreciente en el período, (rho1=0,23 para el año 2001 y 0,14 para el año 2011) y una relación inversa con el gasto en sa- lud por inscrito creciente en el tiempo (rho1=-0,13 $\mathrm{y}-0,2)$.

Para el año 2011 además se observa una correlación positiva a tasa de natalidad $(\operatorname{rhol}=0,13)$ y negativa a la inversión en salud municipal (rho1=$0,21)$ y a la tasa de médicos disponibles (rho1=$0,12)$.

Al incluir en el análisis sólo las comunas que presentaron defunciones infantiles, se observa que la TMI mantiene una correlación positiva a pobreza sólo para el año 2001 (rho2=0,27), desapareciendo la relación el año 2011 (rho2=0,09); mientras que el gasto en salud por inscrito no presenta correlación a TMI.

Con este análisis se revelan además otras asociaciones a mortalidad infantil de magnitud creciente en el período. Así, para ambos años se encuentra una relación inversa creciente entre mortalidad infantil y escolaridad promedio de la población (rho2=-0,16 y -0,24), sumado a una creciente asociación al porcentaje de retiro de la enseñanza media (rho2 $=0,27$ para el 2011).

En relación a características demográficas, se observa una asociación inversa a tamaño de población comunal $(\mathrm{rho} 2=-0,38)$ y tasa de natalidad $(\mathrm{rho} 2=-$ $0,13)$.

Con respecto a características de urbanización, para ambos años se encuentran relaciones inversas, de magnitud creciente, entre TMI y cobertura de agua potable (rho2=-0,15 y -0,28), proporción de población urbana (rho2=-0,12 y -0,22) y densidad de población (rho2=-0,17 y -0,37); mientras que, 
para el año 2011 aparece una relación inversa a la cobertura de alcantarillado en las viviendas (rho2=$0,20)$.

En cuanto a la disponibilidad de recursos financieros e infraestructura de salud municipal, se encuentran correlaciones positivas entre TMI y dependencia del FCM sobre ingresos propios, que mantiene su magnitud durante el período, $($ rho $2=0,15$ y 0,18$)$ y disponibilidad de centros ambulatorios por inscrito, de magnitud creciente $(\mathrm{rho} 2=0,18 \mathrm{y}$ $0,34)$. En tanto, se observan relaciones inversas, de magnitud creciente, a inversión en salud municipal por inscrito (rho2=-0,11 y -0,22) y número de SAPU por cada 10.000 habitantes (rho2=-0,19 y -0,23). Para el año 2011, aparecen además relaciones entre TMI y tasa de médicos por 1.000 inscritos (rho2 $=-0,18)$ y distancia al hospital base de referencia $($ rho $2=0,12)$.

El análisis de correlación múltiple, si bien presenta importantes limitaciones a la inferencia que pueda realizarse, dada la existencia de correlación entre las distintas variables (en algunos casos de alta magnitud), entrega una primera aproximación hacia la construcción de un perfil de características comunales presentes en territorios con mayor mortalidad infantil. En ese sentido, este análisis muestra un cambio temporal en cuanto al perfil de las comunas en su relación a mortalidad infantil, adquiriendo relevancia en esta caracterización, los déficits socioeconómicos, urbanísticos y de disponibilidad de recursos financieros y sanitarios al final del período de análisis, permitiendo plantear, preliminarmente, que la mortalidad infantil pudiese estar persistiendo en comunas con rezagos en su desarrollo.

\section{Evolución de la Desigualdad en Mor- talidad Infantil comunal}

Los distintos indicadores construidos concuerdan en que la desigualdad en mortalidad infantil comunal presenta fluctuaciones durante el período, pero con una clara tendencia al aumento en relación al año 2001. En particular, el coeficiente de Gini de mortalidad infantil aumentó en $11 \%$ entre los años 2001 (0.44) y 2011 (0.49).

En cuanto al ingreso en salud municipal por inscrito, la desigualdad entre comunas es menor a la observada para mortalidad infantil; sin embargo, también presenta un aumento en el período $(47 \%$ en el caso del coeficiente de Gini), con fluctuaciones más pronunciadas a partir del año 2007.

Un análisis de correlación entre la mediana de TMI comunal y el Gini de TMI para el período, mostró que a menor mortalidad infantil existe mayor desigualdad en el indicador a nivel comunal $(\mathrm{rho}=-0.580, \mathrm{p}=0.06)$; mientras que, la correlación entre la mediana de TMI para cada año y el ingreso en salud municipal por inscrito, muestra que a menor ingreso mayor mortalidad infantil a nivel comunal (rho=-0.6727, $\mathrm{p}=0.02$ ).

En tanto, la desigualdad de ingresos en salud municipal se observó positivamente relacionada a este ingreso $(\mathrm{rho}=0.5438, \mathrm{p}=0.08)$.

Finalmente, la correlación entre ambas mediciones de desigualdad resulta directa, aunque no significativa (rho gini $=0.47, \mathrm{p}=0.14$ ), lo cual apuntaría hacia una mayor desigualdad en mortalidad infantil a nivel comunal, a mayor desigualdad de ingresos en salud municipal, sugiriendo que las políticas públicas implementadas para fortalecer la atención primaria de administración municipal, pudiesen estar ahondando la desigualdad estructural en mortalidad infantil.

El análisis de correlación fue verificado con análisis de regresión lineal. El análisis de correlación y las rectas de regresión se muestran en el Gráfico 3. Los coeficientes de regresión obtenidos se muestran en la Tabla 1.

La figura 3 muestra las variaciones observadas entre los años 2001 y 2011 en mortalidad infantil, ingreso en salud municipal por inscrito y sus respectivos coeficientes de Gini, con datos comunales agregados por región.

A nivel regional, se observa que Atacama (III), Los Ríos (XIV) y la Región Metropolitana (RM) presentaron un aumento en la TMI con disminución en la desigualdad comunal, entre los años 2001 y 2011; lo cual implica que, la mortalidad infantil habría aumentado homogéneamente entre sus comunas. En tanto en la región del Maule (VII), se observa un aumento leve tanto en mortalidad infantil como en su desigualdad comunal.

En las regiones de Aysén (XI), Magallanes (XII) y de Arica y Parinacota (XV), tanto la TMI como el coeficiente de Gini se mantuvieron estables. En el resto de las regiones, si bien presentan una disminución de la mortalidad infantil comunal, la desigualdad entre comunas aumentó, salvo para el caso de la región del Biobío, en la cual la desigualdad comunal se mantuvo constante.

En cuanto al ingreso en salud municipal por inscrito (medido en pesos del 2012), se observa un aumento en todas las regiones ${ }^{11}$. Sin embargo, la de-

\footnotetext{
${ }^{11}$ No se dispone del ingreso en salud por inscrito del año 2001 para las comunas de la región de Aysén y Magallanes. En tanto, para las regiones de Tarapacá (I) y Arica y Pari-
} 
Figura 2: Evolución de la desigualdad en Mortalidad Infantil Comunal e ingresos en Salud Municipal. Chile, $2001-2011$

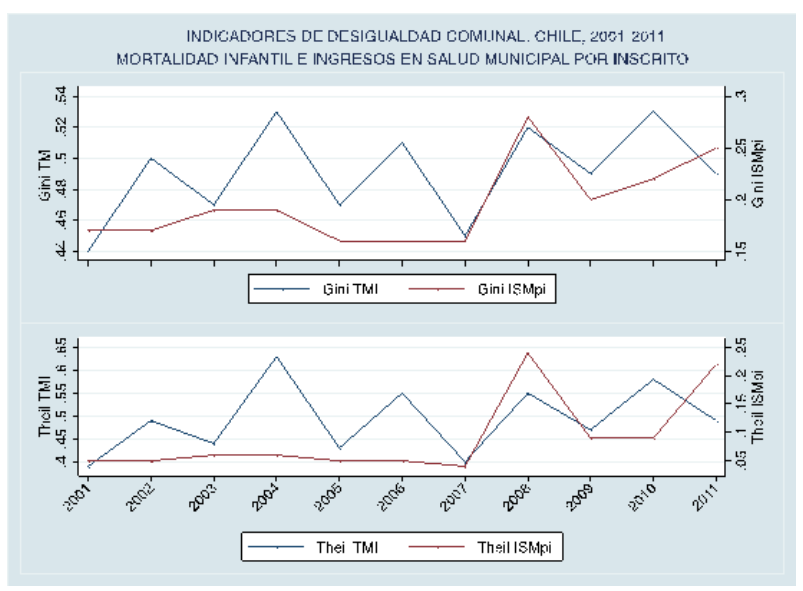

(a) Coeficientes de Gini y Theil

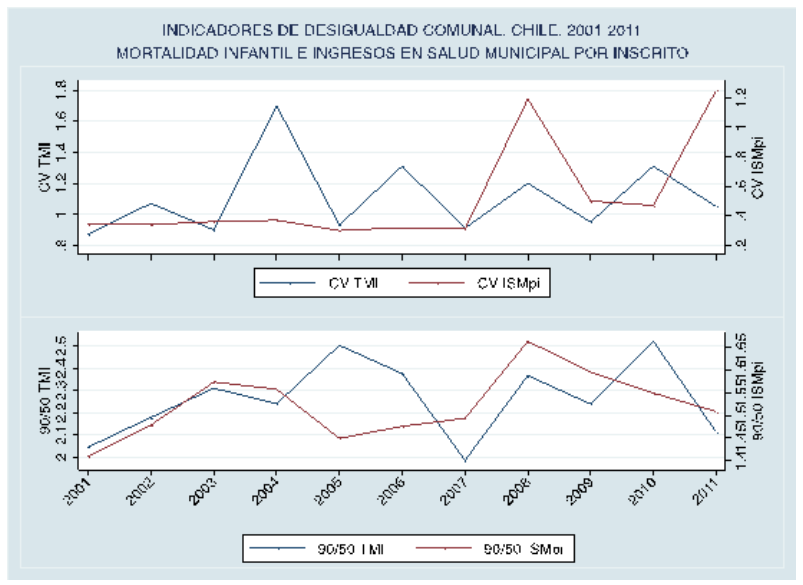

(b) Coeficiente de variación y razón 90/50

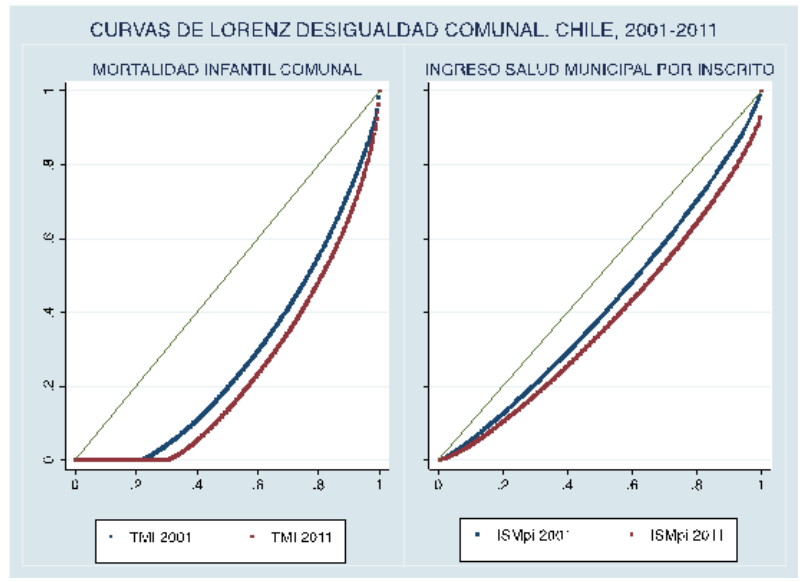

(c) Curvas de Lorenz

TMI: Tasa de Mortalidad Infantil, ISMpi: Ingreso en salud municipal por inscrito 
Tabla 1: Coeficientes de regresión lineal simple

\begin{tabular}{|c|c|c|c|c|}
\hline $\begin{array}{c}\text { Predictor } \\
\text { Predicho }\end{array}$ & TMI & ISMpi & ISMpi & Gini ISMpi \\
\hline Gini TMI & $-0.0468^{* *}$ & & & 0.6159 \\
& $\mathrm{R}^{2}=0.4644$ & & & $\mathrm{R}^{2}=0.2268$ \\
\hline TMI & & $-0.0270^{* *}$ & & \\
& & $\mathrm{R}^{2}=0.5188$ & & \\
\hline $\begin{array}{c}\text { Gini } \\
\text { ISMpi }\end{array}$ & & & $0.0021^{* *}$ & \\
\hline
\end{tabular}

TMI: Tasa de Mortalidad Infantil, ISMpi: Ingreso en salud municipal por inscrito.

**Nivel de confianza: 95

Figura 3: Relaciones entre Mortalidad Infantil Comunal

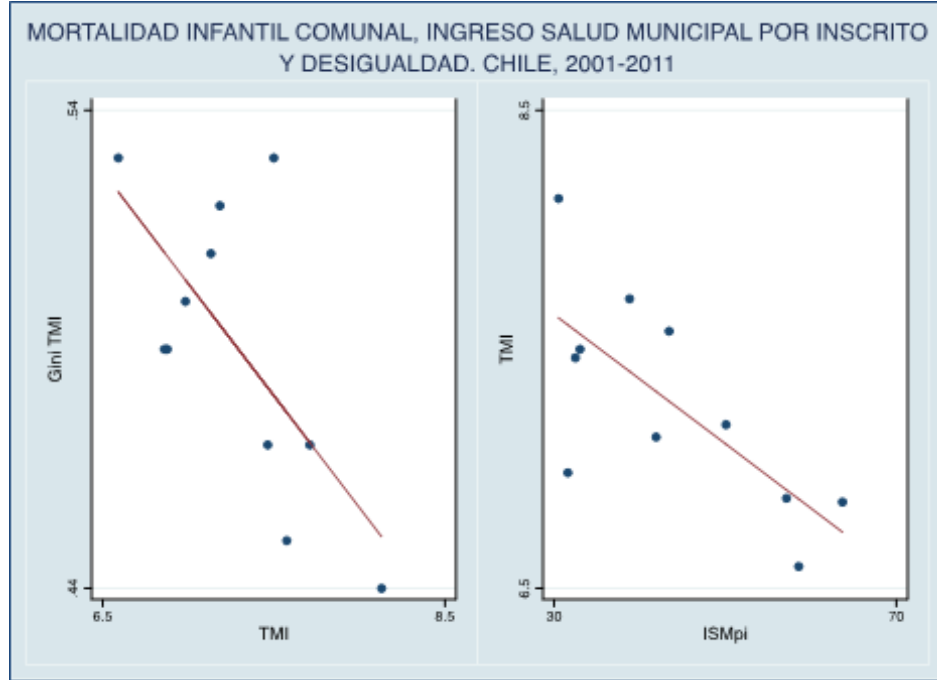

MORTALIDAD INFANTIL COMUNAL, INGRESO SALUD MUNICIPAL POR INSCRITO Y DESIGUALDAD. CHILE, 2001-2011
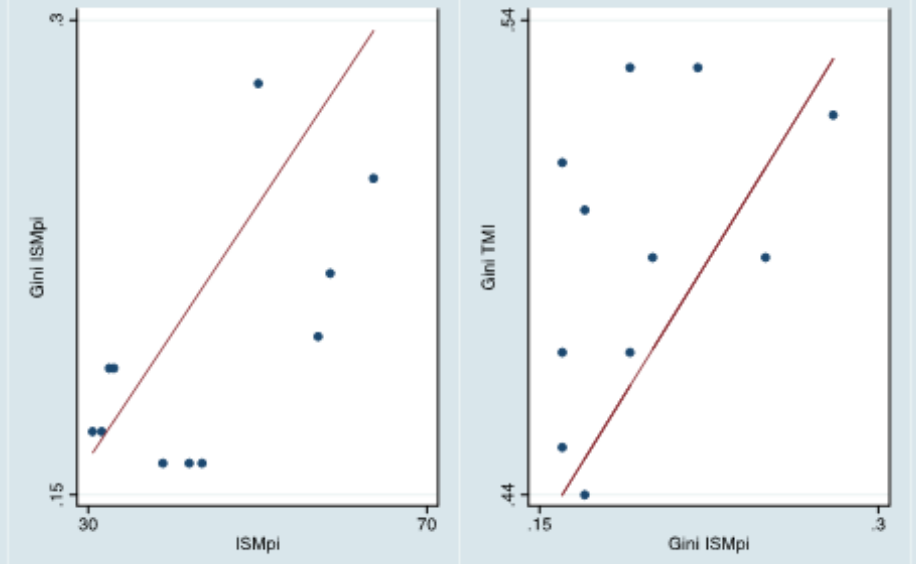

TMI: Tasa de Mortalidad Infantil, ISMpi: Ingreso en salud municipal por inscrito 
sigualdad de este indicador aumentó en 6 de las 11 regiones con datos disponibles.

\section{Análisis de panel Mortalidad infantil comunal, $2001-2011$}

La Tabla 2 presenta una síntesis de los principales coeficientes de regresión en distintos modelos construidos con el panel de datos del período, de acuerdo a la expresión (1) de modelos con efectos aleatorios $^{12}$, donde $i$ representa las comunas y $t$ los años evaluados:

$$
T M I_{i t}=\alpha+\beta X_{i t}+\mu_{i}+\epsilon i t
$$

El primer modelo evaluado muestra que la mortalidad infantil se encontraría explicada por mayor tasa de pobreza comunal (significativa al 1\%), mayor distancia al hospital de referencia (significativa al $5 \%$ ), menor inversión en salud municipal por inscrito (significativa al 1\%) y en comunas con mayor población comunal (significativa al $10 \%$ ).

Sin embargo, este primer modelo muestra una asociación directa a años de escolaridad (aunque no significativa), lo cual estaría en relación contraria a lo planteado en la literatura. Esto se podría explicar por la significativa correlación inversa encontrada para pobreza y escolaridad (rho -0.5), lo cual puede generar problemas de multicolinealidad en el modelo.

Para evaluar este efecto, en los siguientes modelos se realizó un ordenamiento descendente de las comunas según pobreza (mayor ranking a mayor pobreza) y ascendente según escolaridad promedio de la población (mayor ranking a menor escolaridad). Con esto, se observa un efecto negativo de la menor educación sobre la mortalidad infantil (significativa al $5 \%$ para todos los modelos), manteniendo los efectos encontrados en pobreza, población comunal, distancia al hospital base e inversión en salud municipal.

Con estas especificaciones, se evidencia que la mortalidad infantil comunal estaría explicada por una menor cobertura de agua potable, un menor gasto en salud municipal por inscrito, una menor tasa de médicos y no contar con SAPU en la comuna (para la mayoría de los modelos con nivel de confianza entre $90 \%$ y $99 \%$ ).

Los coeficientes fueron ajustados por heteroce-

nacota (XV) no se disponen de suficientes datos comunales para el cálculo del coeficiente de Gini.

${ }^{12}$ Considerando efectos aleatorios, según lo avalado por Test de Hausman. dasticidad, comprobando además que no existiera autocorrelación serial en cada modelo. Finalmente, se realizó un análisis de componentes principales para determinar perfiles comunales en relación a las variables socioeconómicas, sanitarias y municipales evaluadas. Este análisis permitió identificar 3 perfiles generales de comunas, que explican en conjunto el $65 \%$ de la varianza:

- Bueno: corresponde a comunas con menor tasa de pobreza, alta escolaridad promedio de la población, alta cobertura de servicios básicos, mayor tamaño poblacional y proporción de población urbana, menor dependencia al FCM sobre ingresos propios y con disponibilidad de SAPU. Ejemplos: Las Condes, Vitacura, La Reina, Providencia y Ñuñoa (Región Metropolitana), Viña del Mar (V Región), Antofagasta (II Región).

- Malo: comunas con alta tasa de pobreza, menor escolaridad promedio de la población, menor gasto municipal en asistencia social per cápita, alta dependencia al FCM, menores coberturas de servicios básicos, menor población comunal y urbana. Ejemplos: Lonquimay (IX Región), Panguipulli y Río Bueno (XIV Región), Combarbalá y Los Vilos (IV Región), Coelemu (VIII Región), Chañaral (III Región).

- Mayores recursos sanitarios: principalmente, comunas con alta inversión municipal por inscrito en el sistema de atención primaria y secundariamente, con una menor distancia al hospital base de referencia. Ejemplos: Mariquina (XIV Región), Ancud (X Región), Rengo (VI Región), Lumaco, Reinaco y Angol (IX Región), Lo Barnechea (Región Metropolitana).

La Tabla 3 muestra la mediana de características comunales entre los años 2001 y 2011 en tres comunas del país que ejemplifican los perfiles construidos: Las Condes, Lonquimay y Lumaco, respectivamente.

A continuación se evaluó un modelo de regresión con datos de panel para estos componentes, confirmando que comunas con perfil socioeconómico "bueno" y mayores recursos sanitarios tendrían menor mortalidad infantil (disminución de TMI en $24,2 \%$ y $23,7 \%$, respectivamente); mientras que, las comunas con perfil "malo" presentarían mayor mortalidad infantil (aumento de TMI en $41 \%$ ). Todos los coeficientes resultan significativos a un nivel de confianza de $99 \%$. Los resultados de este análisis se presentan en la Tabla 4.

Con el fin de evidenciar la realidad en mortalidad infantil de las distintas comunas del país y observar 
Figura 4: Evolución de la desigualdad en Mortalidad Infantil Comunal e ingresos en Salud Municipal. Chile, $2001-2011$

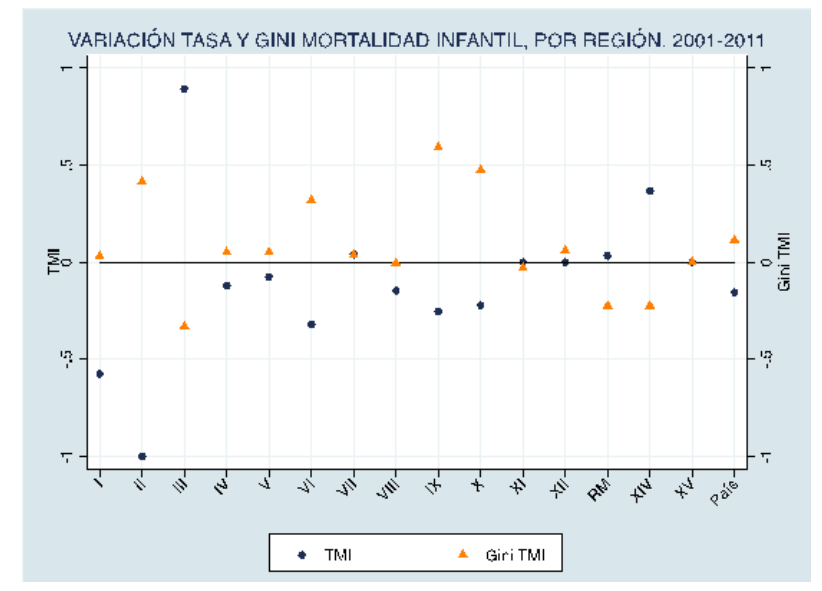

(a) Tasa y Gini de mortalidad infantil

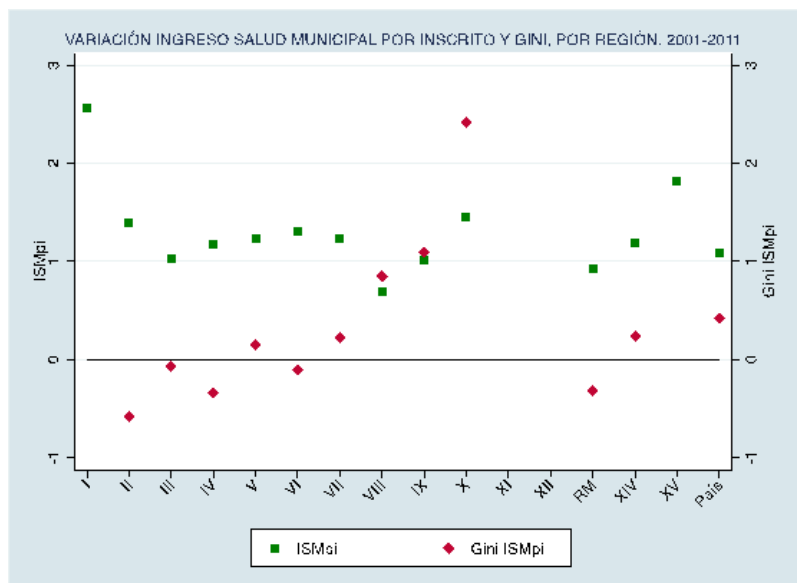

(b) Ingreso en salud municipal por inscrito y Gini

TMI: Tasa de Mortalidad Infantil, ISMpi: Ingreso en salud municipal por inscrito

Tabla 2: Resumen coeficientes de estimación de tmi por panel, efectos aleatorios

\begin{tabular}{llll}
\hline Variables & $\mathbf{( 1 )}$ & $\mathbf{( 3 )}$ & $\mathbf{( 5 )}$ \\
\hline \hline $\begin{array}{l}\text { pobreza } \\
\text { ranking pobreza }\end{array}$ & $0.0839^{*+*}$ & $0.0076^{* * *}$ & $0.0071^{* * *}$ \\
escolaridad promedio & 0.1816 & & \\
ranking educación & & $-0.0082^{* *}$ & $-0.0075^{* *}$ \\
agua potable & -0.0154 & $-0.0283^{* * *}$ & $-0.0272^{* *}$ \\
población comunal & $0.3 \mathrm{e}^{5 *}$ & $0.3 \mathrm{e}^{* 5^{* *}}$ & $0.3 \mathrm{e}^{-5 * *}$ \\
dependencia FCM & -0.0097 & & \\
gasto salud & -0.0041 & $-0.0086^{*}$ & $-0.0087^{*}$ \\
inversión salud & $-0.1214^{* * *}$ & $-0.1177^{* * *}$ & $-0.1204^{* * *}$ \\
SAPU & -0.4048 & $-0.6232^{* *}$ & $-0.5816^{* *}$ \\
médicos & -0.1258 & $-0.1395^{* *}$ & -0.1324 \\
distancia hospital & $0.0078^{* *}$ & $0.0075^{* *}$ & $0.0072^{* *}$ \\
constante & $8.768^{* * *}$ & $12.535^{* * *}$ & $12.259^{* * *}$ \\
\hline
\end{tabular}

TMI: Tasa de Mortalidad Infantil, ISMpi: Ingreso en salud municipal por inscrito 
la variación de la TMI entre el año 2001 y 2011, a continuación se presentan los mapas construidos con intervalos de la mediana de mortalidad infantil comunal para el país y detallando por región.

El conjunto de resultados expuestos en este trabajo muestran que la mortalidad infantil sigue estando fuertemente vinculada tanto a la realidad socioeconómica de las comunas, como a la disponibilidad de recursos sanitarios, principalmente, de los recursos municipales destinados a salud. Por una parte, existe un grupo de comunas cuyas altas tasas de mortalidad infantil para el período 2001-2011 podrían explicarse por el conjunto de limitaciones socioeconómicas de su población; en tanto, se observan comunas que a pesar de tener estas limitaciones, logran obtener bajas tasas de mortalidad infantil en relación a la mediana nacional, gracias a una mayor disponibilidad de recursos en salud municipal, principalmente, en cuanto a inversión por población inscrita.

En este sentido, los resultados presentados apuntan a que la disparidad en cuanto a la realidad socioeconómica y de recursos sanitarios observada en las comunas del país, podría explicar la desigualdad observada en mortalidad infantil a nivel comunal en el período.

\section{Conclusiones}

En los últimos años el país ha experimentado un crecimiento acelerado y condiciones de estabilidad económica, destacándose entre los países de la región. Esto sin duda puede generar buenas perspectivas en todos los ámbitos que influyen en el bienestar de la población, incluida la salud. Es de esperar que en este sentido los avances económicos continúen generando un buen aporte a los distintos indicadores sanitarios, como la esperanza de vida al nacer, la mortalidad general, materna e infantil; indicadores que Chile ha lucido con orgullo por varias décadas.

Es reconocido que el éxito alcanzado en esta materia desde mediados del siglo XX, se debió a la intervención de políticas públicas sanitarias estrechamente vinculadas a mejoras socio-económicas de la población, impulsadas por la generación de médicos de la llamada Medicina Social. El programa materno-infantil es un claro ejemplo de ello. Hacia finales del siglo XX, surgieron nuevas políticas bajo este enfoque, fortaleciéndose el Programa de Salud del Niño con la creación de las Salas de Infecciones Respiratorias Agudas (Salas IRA), las campañas de invierno y los servicios de atención primaria de urgencias (SAPU). Con el desarrollo alcanzado por estas políticas a lo largo del siglo, la mortalidad infantil a nivel país logró un descenso de tal aceleración, no observada en ningún otro país de América Latina, y un cambio en el perfil epidemiológico de las defunciones infantiles.

Así como el crecimiento económico es fundamental para el desarrollo de una sociedad, lo es también la forma en que este se distribuya en su interior. Desde distintos sectores se ha planteado el dilema de crecer en desigualdad que se observa en Chile. Diversos autores han evidenciado la alta desigualdad de ingresos y la falta de equidad (de acceso y resultados) en ámbitos centrales para el desarrollo de capital social, como la educación o el empleo. Sin embargo, poco se ha investigado acerca del efecto de crecer en desigualdad socio-económica sobre la desigualdad de acceso y resultados en salud, bajo un enfoque de oportunidades básicas. Poco reveladores para ello resultan los indicadores sanitarios nacionales, los cuales pueden estar ocultando disparidades relevantes a niveles sub-nacionales.

En este sentido, este estudio buscó explorar la realidad de la mortalidad infantil a nivel comunal y su distribución. Los resultados muestran que detrás de las exitosas cifras promedio se esconde una gran dispersión entre comunas, generando una desigualdad en aumento, que alcanza al año 2011 un Gini de mortalidad infantil comunal de 0.49 y que se correlaciona altamente a la desigualdad encontrada para la distribución de ingresos en salud municipal por población inscrita. Ciertas características municipales y de la población comunal resultan ser factores explicativos de esta desigualdad, principalmente, la tasa de pobreza, la escolaridad promedio de la población y el acceso a ciertos recursos sanitarios, como contar con SAPU, estar a menor distancia del hospital de referencia o pertenecer a un municipio que puede destinar más recursos para gasto e inversión en salud.

La mayoría de los estudios sobre mortalidad infantil la han vinculado a condiciones propias del niño, como su peso de nacimiento o las semanas de gestación, o a condiciones socio-económicas de su entorno inmediato, principalmente, de su madre. ¿Cómo se podría explicar entonces que la mortalidad infantil en una comuna pudiese estar asociada a las características de la población en su conjunto y a la holgura de sus finanzas municipales? Planteamientos plausibles para resolver esta pregunta son la segregación social y la alta vulnerabilidad que puede generar que la administración de la atención primaria de salud (y en cierta parte, su financiamiento) sea responsabilidad de los municipios, al alero de sus grandes disparidades en cuanto a la disponibilidad de recursos. 
Figura 5: Mapas de Mortalidad Infantil Comunal. Chile, 2001 y 2011

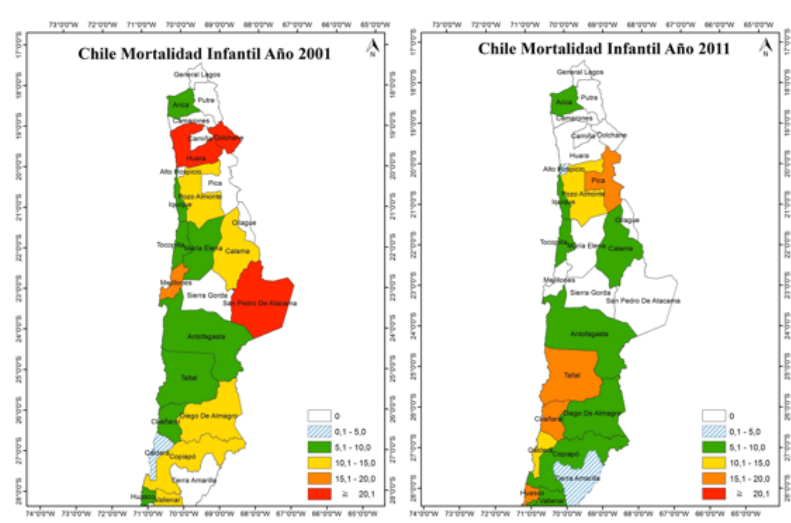

(a)

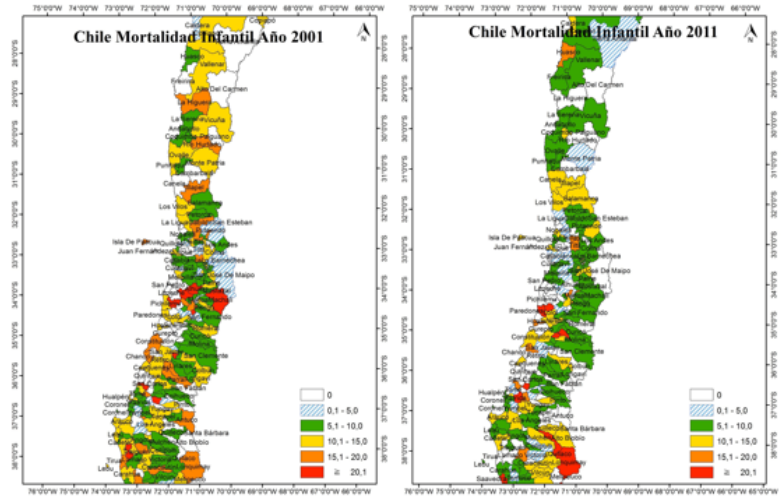

(b)

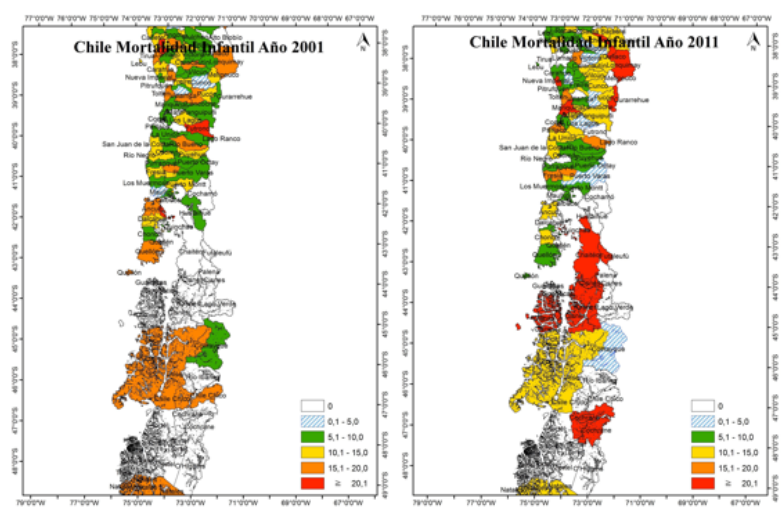

(c)

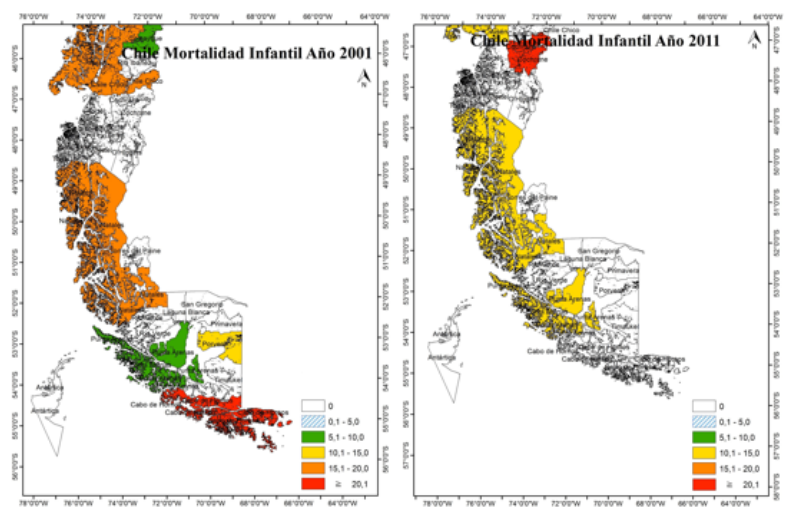

(d)

Fuente: Andrés Martínez Muñoz, Egresado de Geografía de la Universidad de Chile

Rev. Est. de Políticas Públicas, 2015, 2(noviembre) 
Tabla 3: Características comunales*. Chile, 2001-2011

\begin{tabular}{|c|c|c|c|c|}
\hline $\begin{array}{ll}\begin{array}{l}\text { Caract. } \\
\text { comunales }\end{array} & \text { Perfil } \\
\end{array}$ & $\begin{array}{l}\text { Bueno } \\
\text { LAS CONDES } \\
\text { (RM) }\end{array}$ & $\begin{array}{l}\text { Malo } \\
\text { LONQUIMAY } \\
\text { (IX) }\end{array}$ & $\begin{array}{l}\text { Rec. Salud } \\
\text { LUMACO } \\
\text { (IX) }\end{array}$ & CHILE \\
\hline Pobreza (\%) & 2.3 & 25.7 & 36.8 & $\bar{~}$ \\
\hline Escolaridad promedio (años) & 13.2 & 6.8 & 5.6 & 8.1 \\
\hline Alcantarillado (\%) & 89.0 & 46.5 & 51.9 & 68.8 \\
\hline Agua Potable (\%) & 89.0 & 35.0 & 36.5 & 71.3 \\
\hline Población comunal (hab.) & 274035 & 11096 & 10857 & 17459 \\
\hline Población urbana $(\%)$ & 100 & 33.6 & 36.8 & 63.8 \\
\hline Dependencia al FCM (\%) & 2.3 & 90.1 & 93.2 & 68.3 \\
\hline $\begin{array}{l}\text { Gasto municipal en asistencia } \\
\text { social per cápita }(\$)\end{array}$ & 10971 & 1198 & 1750 & 1430 \\
\hline $\begin{array}{r}\text { Gasto municipal anual en salud } \\
\text { por inscrito (\$) }\end{array}$ & 98068 & 47004 & 49710 & 42983 \\
\hline $\begin{array}{r}\text { Inversión municipal anual en } \\
\text { salud por inscrito (\$) }\end{array}$ & 1374 & 65 & 3803 & 22 \\
\hline $\begin{array}{r}\text { Tasa de centros cada mil } \\
\text { inscritos }\end{array}$ & 0.03 & 1.4 & 0.7 & 0.3 \\
\hline Disponibilidad de SAPU & SI & NO & NO & $48.7 \%$ \\
\hline $\begin{array}{r}\text { Tasa de médicos cada mil } \\
\text { inscritos }\end{array}$ & 0.7 & 0.2 & 0.1 & 0.2 \\
\hline $\begin{array}{r}\text { Distancia al hospital base de } \\
\text { referencia }(\mathbf{k m})\end{array}$ & 11 & 120 & 28 & 17 \\
\hline $\begin{array}{r}\text { Tasa de Mortalidad Infantil } \\
\text { cada } 1000 \text { nacidos vivos }\end{array}$ & 4.8 & 16.1 & 6.0 & 7.2 \\
\hline
\end{tabular}

*Mediana de las variables para el período 2001-2011.

Fuente: Elaboración propia

Tabla 4: Estimación de TMI para perfiles comunales. Panel 2001-2011

\begin{tabular}{cccc}
\hline Perfil & Coef. & $\mathbf{z}$ & $\mathbf{P}>|\mathbf{z}|$ \\
\hline \hline Bueno & -0.2424714 & -3.49 & $<0.001$ \\
Malo & 0.4097104 & 2.87 & 0.004 \\
Recursos salud & -0.2371555 & -3.16 & 0.002 \\
Constante & 8.369858 & 63.72 & $<0.001$ \\
\hline sigma u & 1.4555 & & \\
sigma e & 6.43547 & & \\
rho & .04867 & & \\
R2 within & 0.0036 & \\
R2 between & 0.0620 & \\
R2 overall & 0.0124 & \\
Obs. & 2712 & \\
* Modelo de efectos aleatorios (Test de Hausman: prob>chi2=0.1476), descartando autocorrelación (Test de \\
Wooldridge: prob $>\mathrm{F}=0.3236$ ) y ajuste de coeficientes por heterocedasticidad.
\end{tabular}

Fuente: Elaboración propia 
No es objetivo de este estudio testear estas hipótesis y menos aún esbozar soluciones de políticas públicas para resolver estas disparidades. El solo tema de la administración municipal de salud abre un sin número de aspectos y posturas a debatir, entre enfoques de descentralización por elección pública o centralizadores por teoría de agenteprincipal.

Estos resultados reflejan la desigualdad que existe en Chile en cuanto a la oportunidad de sobrevivir durante el primer año de vida. Que la sobrevida de un niño pueda estar aún determinada por las condiciones socio-económicas del territorio donde nace, plantea además de un dilema ético, un llamado de atención sobre la necesidad de evaluar la distribución de los recursos públicos en salud, revisar el enfoque de las políticas públicas para la disminución de la mortalidad infantil (centradas en la actualidad, principalmente, en mejorar la sobrevida de niños prematuros y con malformaciones congénitas, apuntando a disminuir el indicador a nivel país) y sobre la necesidad de retomar el enfoque socio-sanitario de la bien llamada Medicina Social de mediados del siglo XX, cuyas políticas públicas contarían con plena vigencia en la resolución de las necesidades de salud que hoy tiene la población de buena parte de nuestro territorio.

\section{Referencias}

Albala, C., Vio, F., Robledo, A., e Icaza, G. (1993). La transición epidemiológica en Chile. Revista Médica de Chile, 121:1446-1455.

Castañeda, T. (1985). Determinantes del descenso de la Mortalidad Infantil en Chile: 1975-1982. Cuadernos de Economía, 22(66):195-214.

CELADE. (2009). Proyección de población, américa latina y el caribe. Observatorio Demográfico, $6(7)$.

Chile, G. D. (2005). Los Objetivos De Desarrollo Del Milenio. Primer Informe del Gobierno de Chile. Santiago de Chile.

Donoso, E. (2004). Desigualdad en mortalidad infantil entre las comunas de la provincia de Santiago. Revista Médica de Chile, 132:461-466.

Flores, M. y Cerda, J. (2012). Evolución de la desigualdad en la mortalidad infantil. Archivos de Pediatría del Uruguay, 83(2):139-143.

Jiménez, J. y Romero, M. (2007). Reducción de la mortalidad infantil en Chile: un éxito en dos etapas. Rev. Panam Salud Pública, 21:251-3.
Jiménez de la Jara, J. (2001). Mística, ciencia y política en la construcción de sistemas de salud. la experiencia de chile. Salud Pública Mex, 43:485-493.

Kaempffer, A. y Medina, E. (2006). Mortalidad infantil reciente en Chile: éxitos y desafíos. Rev Chilena de Pediatría, 77(5):492-500.

Kliksberg, B. (2005). América Latina: La región más desigual de todas. Revista de Ciencias Sociales, 11(3):411-421.

Ministerio De Salud Gobierno De Chile (2010). Mortalidad infantil y sus componentes, por Región y Comuna de residencia de la madre. Departamento De Estadísticas E Información En Salud, Santiago de Chile.

Organización Panamericana De La Salud (2003). Atención primaria de salud en las Américas: Las enseñanzas extraídas a lo largo de 25 años y los retos futuros. Washington, DC.

Organización Panamericana De La Salud (2005). Renovación de la Atención Primaria de Salud en las Américas. Washington, DC.

Pan American Health Organization (2002). Health in the Americas. Washington, DC.

Pérez, S., Bárcena, E., y Blanco, M. (2012). Fluctuaciones económicas y mortalidad infantil. XIV Reunión Económica Mundial.

Raña, J., Ferrer, J. C., y Bedregal, P. (2007). Modelo de asignación de recursos en atención primaria. Revista Médica de Chile;, 135:54-62.

Salud, O. P. D. L. (2003). La transición hacia un nuevo siglo de salud en Las Américas. Informe anual de la Directora de la OPS, Washington, DC.

Sánchez, H. y Albalá, C. (2004). Desigualdades en salud: mortalidad del adulto en comunas del Gran Santiago. Revista Médica de Chile, 132:453-460.

Silva, L. y Durán, E. (1990). Mortalidad infantil y condiciones higiénico-sociales en las américas. un estudio de correlación. Rev. Saúde pública, 24(6).

Sistema De Información De Tendencias Educativas En América Latina (2013). Desarrollo en América Latina 2000-2010: Resumen Estadístico Comentado.

Starfield, B. y Shi, L. (2002). Policy relevant determinants of health: an international perspective. Health Policy, 60:201-218. 
Starfield, B., Shi, L., y Macinko, J. (2005). Contribution of primary care to health systems and health. The Milbank Quarterly, 83(3).

Szot, J. (2002). Mortalidad infantil e indicadores económicos en Chile: 1985-1999. Revista Médica de Chile, 130(1):107-112.

Urriola, R. (2007). Alcances para el análisis de la equidad en los sistemas de salud. Cuadernos Médicosociales, 47(2):112-125.
Valdivia, G. (2006). Transición epidemiológica: la otra cara de la moneda. Revista Médica de Chile, 134:675-678.

Vargas, V. y Wasem, J. (2006). Risk adjustment and primary health care in chile. Croatian Medical Journal, 47:459-468. 